\title{
Diagnostik, Therapie und Prävention der Influenza
}

T. Schaberg

(Virusgrippe)

\author{
Diagnosis, Therapy, and Prevention of Influenza
}

\section{Pathophysiologie und Epidemiologie}

Influenza-Virusinfektionen, die zur Virusgrippe führen, bedingen weltweit jedes Jahr eine erhebliche Mortalität und Morbidität. Dabei nimmt die Influenza wegen ihres besonderen Potentials, epidemische Infektionen zu erzeugen, eine Sonderstellung unter den akuten respiratorischen Erkrankungen ein. Der Verlauf einer Virusgrippe wird im Wesentlichen bestimmt durch die Virulenz bzw. Pathogenität des Virus auf der einen Seite und auf der anderen Seite durch die allgemeine und spezifische Immunkompetenz der betroffenen Person. Die spezielle Pathophysiologie der Virusgrippe ist recht gut bekannt [1]. Die Infektionsübertragung erfolgt in der Regel auf dem Luftwege mittels eines Aerosols, das beim Husten oder Niesen von erkrankten Personen abgegeben wird. Aerosolpartikel von weniger als $10 \mu \mathrm{m}$ werden dann in der Regel über die Hände auf die Schleimhäute des Respirationstraktes oder die Konjunktiven übertragen. Eine Inhalation der Aerosole ist jedoch ebenso möglich.

Trifft das Virus auf eine immunologisch naive Person, so dauert die Produktion von spezifisch gegen das Influenza-Virus gerichteten T-Zellen in der Regel 1 - 2 Tage. Spezifische Immunglobuline der IgA-, IgM- und IgG-Klasse lassen sich in der Regel erst nach Abklingen des akuten Krankheitsbildes nachweisen. Diese vermitteln also einen immunologisch-kompetenten Schutz nur bei einer erneuten Infektion mit Viren, die identische oder nahe verwandte Antigenstrukturen haben [1,2].

Influenzaviren gehören zur Familie der Orthomyxoviren und gliedern sich in die Gruppe der Influenza A- und Influenza B-Viren. Auf der Oberfläche der Viren befinden sich Hämagglutinine und Neuraminidasen. Hämagglutinine und Neuraminidasen unterliegen einer ständigen Veränderung ihres molekularen Auf- baus. Da sie für das Immunsystem die wichtigsten antigenen Strukturen darstellen, bedingt die Veränderung dieser Moleküle immer neue Kombinationen von Antigenstrukturen [2]. Geringe Strukturänderungen, die kontinuierlich auftreten, werden als Antigendrift bezeichnet, der dem Immunsystem eine partielle Wiedererkennbarkeit antigener Struktur erlaubt und somit eine Teilimmunität hinterlässt, die jedoch abhängig vom Verbreitungsgebiet der Virustypen die jährlich im Winterhalbjahr auftretenden Epidemien nicht völlig verhindern kann. Ausgeprägtere Änderungen bezeichnet man als Antigenshift. Hierbei ist die Änderung der Antigenstruktur so umfassend, dass sie dem Immunsystem vollkommen unbekannt ist. Solche Änderungen treten im Verlauf mehrerer Jahre bis Jahrzehnte auf und führen zu neuen, oft weltweiten Pandemien, da sie auf ein vollkommen naives humanes Immunsystem treffen [2,3].

Während die Grippeviren einerseits die erhebliche Morbidität weltweit induzieren, sind die bakteriellen Superinfektionen außerhalb von Pandemien im Wesentlichen für die zusätzliche Mortalität verantwortlich [4]. Influenza-Viren sind in der Lage, das Epithel der Atemwege lytisch zu zerstören und darüber hinaus eine passagere Immunsuppression durch die Verminderung der Zahl durch der in der Mukosa vorhandenen Makrophagen und T-Lymphozyten zu erzeugen. Auf dem Boden der durch eine Zell-Lyse geschädigten Schleimhaut und der Immunitätsverminderung kann es sehr leicht zu bakteriellen Superinfektionen in den tiefen Atemwegen und im Lungenparenchym kommen. Die führenden Erreger sind hierbei Streptococcus pneumoniae, Haemophilus influenzae und Staphylococcus aureus. Betroffen sind hiervon in erster Linie sehr junge Menschen unterhalb des 4. Lebensjahres und ältere Menschen oberhalb des 60. Lebensjahres. Primäre Pneumonitiden, bedingt durch das Grippevirus selbst, sind Erkrankungen, deren Verlauf sehr variabel ist

Serienherausgeber: S. Ewig, T. Schaberg

Institutsangaben Zentrum für Pneumologie, Diakoniekrankenhaus Rotenburg

Korrespondenzadresse Prof. Dr. T. Schaberg · Zentrum für Pneumologie · Diakoniekrankenhaus Rotenburg · Verdener Str. 200 · 27356 Rotenburg ·E-mail: schaberg@diako-online.de 
und vom bloßen Zufallsbefund bis zur Entwicklung eines ARDS reicht. Weitere Komplikationen einer Infektion mit Grippeviren können Myokarditiden mit malignem Verlauf und ZNS-Infektionen mit Meningitiden und Encephalitiden darstellen.

In der Saison 2000/2001 wurde das erste Grippevirus in Deutschland in der 47. Kalenderwoche bei einem Berliner Patienten isoliert [5]. Erst ab Januar 2001 kam es zu einer Ausbreitung der Erkrankung über die Region Berlin-Brandenburg hinaus. Der Höhepunkt der Grippemorbidität wurde in Deutschland im Beginn des Jahres 2001 in der 4. und 5. Woche erreicht. In diesem Zeitraum konnten $75 \%$ aller in Deutschland während des Winters 2000/2001 isolierten Influenza-Viren nachgewiesen werden.

Primär für die Epidemie des Jahres 2000/2001 verantwortlich waren Influenza A-Viren, der Anteil der Influenza B-Virusinfektionen lag bei $4,5 \%$. Diese wurden jedoch insbesondere gegen Ende der Epidemie nachgewiesen.

Daten über die Verbreitung der Influenza-Virusinfektion werden in Deutschland durch ein Surveillance-System erhoben [6]. Dies beruht auf der Arbeitsgemeinschaft Influenza, der etwa 530 über Deutschland verteilte Arztpraxen angehören. Diese Arztpraxen melden in der Influenza-Saison wöchentlich den Anteil der akuten respiratorischen Infektionen (ARI), die in den einzelnen Praxen diagnostiziert worden sind. Aus langjähriger Erfahrung ist belegt, dass der Verlauf der akuten respiratorischen Infektionen sehr gut mit dem Verlauf der Influenza-Epidemie übereinstimmt. Ein Anstieg der Rate der ARI über einen Schwellenwert hinaus zeigt sehr deutlich den Beginn der Grippevirus-Epidemie an. Grippeepidemien verlaufen in der Regel nach einem typischen Muster, das am Beginn eine Zunahme von akuten Atemwegsinfektionen bei Kindern zeigt. Nach diesem ersten Zeichen der Epidemie findet sich eine erhöhte Erkrankungsrate vorwiegend in Institutionen, wo größere Gruppen von Risikopatienten zusammen leben, wie z.B. in Seniorenheimen. Innerhalb eines Zeitraumes von 2-4 Wochen breitet sich die Infektion dann in der Regel innerhalb der Bevölkerung aus. Der Gipfel der Epidemie hält in der Regel 4-6 Wochen an, dann kommt es zum langsamen Abflauen der Krankheitswelle.

Darüber hinaus werden von Praxen auch Rachenabstriche angefertigt und in verschiedenen Referenzzentren untersucht. Hier spielt einerseits das nationale Referenzzentrum für Influenza-Viren in Hannover und andererseits das Robert Koch-Institut in Berlin eine führende Rolle. Die Aufgabe des nationalen Referenzzentrums für die Influenza besteht in der Typisierung und Charakterisierung der angezüchteten Influenza-Stämme, der Bestimmung ihrer Subtypen und Varianten sowie in der Charakterisierung der Oberflächenantigene Hämagglutinine und Neuraminidasen mittels Antiseren, Polymerase-Kettenreaktion und Genom-Analyse [7].

Nach dem neuen Infektionsschutz-Gesetz ist der direkte Nachweis von Influenza-Viren meldepflichtig. Auf diese Weise erhält das RKI im Zusammenhang mit den Daten des nationalen Referenzzentrums für Influenza ein gutes Bild über die aktuelle epidemiologische Situation.
Der oben skizzierte Epidemieverlauf gilt allerdings nicht für Viren mit erheblichem Antigenshift, die auf ein immunologisch naives humanes Immunsystem treffen. Epidemien mit solchen Viren können jederzeit zu Pandemien führen, die in der Vergangenheit eine erschreckend Zahl von Opfern gefordert haben. Allein im abgelaufenen Jahrhundert gab es drei weltweite Pandemien: 1918-1920 die Spanische Grippe (Influenza Virus A-H1N1), der 20-50 Millionen Menschen weltweit zum Opfer fielen; 1957-1960 die Asiatische Grippe (Influenza A-H2N2) und 1968-70 die Hongkong-Grippe (Influenza A-H3N3) mit jeweils zirka 1 Millionen Todesopfer [3].

Die Vorhersage einer neuen Pandemie ist bisher bedauerlicherweise nicht möglich. Es muss aber unverändert mit dieser Möglichkeit gerechnet werden.

Aus diesem Grund hat eine Arbeitsgruppe des Robert Koch-Institutes Anfang des Jahres 2002 berechnet, dass bei einer neuen Pandemie in Deutschland mit 20-25 Millionen Erkrankten gerechnet werden muss, von denen 200000 Patienten wegen der Grippe hospitalisiert werden müssen. Insgesamt kann mit 120000 Grippe-bezogenen Todesfällen gerechnet werden und mit einer jährlichen Exzessmortalität von zirka 175000 Personen [8]. Allein antibiotikapflichtige bakterielle Superinfektionen werden im Falle einer Pandemie in Deutschland bei 1,2 Millionen Patienten erwartet.

Einer Pandemie zu begegnen, wird sich auch in Deutschland als schwierig erweisen.

Die beiden einzigen in Deutschland den Grippeimpfstoff produzierenden Firmen sind zwar in der Lage 3-4 Millionen Impfdosen innerhalb von 3 Monaten nach Eingang des Saat-Stammes zu produzieren; da jedoch mit einer europaweiten Verbreitung der Infektion gerechnet werden muss, reduziert sich die Zahl der effektiv für Deutschland zur Verfügung stehenden Impfdosen primär auf 1 Million. Innerhalb von 4 Monaten könnten maximal weitere 4-5 Millionen Impfdosen und innerhalb von 5 Monaten 7-10 Millionen Impfdosen produziert werden. Auch nach einem Jahr stände allenfalls für die Hälfte der Bevölkerung eine Impfdosis zur Verfügung. Insofern wird man im Falle einer erneuten Pandemie die Impfdosen nach einem spezifischen System abgeben müssen. Nach den Katastrophenplänen des RKI würden zuerst Personen, die im Gesundheitswesen arbeiten, sowie Feuerwehrleute, Polizisten und Personen, die in der Energie- und Wasserversorgung tätig sind, geimpft. Im weitern Verlauf der Epidemie müsste eine Impfstoffabgabe an die Hoch-Risikogruppen (ältere, chronisch kranke Personen sowie Kinder und Jugendliche) erfolgen. Die dritte dann zu berücksichtigende Gruppe umfasst alle Personen, die aufgrund ihres Lebensstils oder ihrer Beschäftigung mit vielen anderen Personen in Kontakt kommen. Dies sind vor allem die Mitarbeiter und Mitarbeiterinnen des öffentlichen Dienstes, die viele Personenkontakte haben, sowie Lehrer, Studenten und Schüler.

Allein um die erstgenannte Gruppe in Deutschland ausreichend gut zu impfen, werden am Beginn einer Epidemie mehr als 7 Millionen Impfdosen gebraucht. Für die Impfung der weiteren genannten Gruppen müssten noch einmal 26 Millionen Impfdosen bereitgestellt werden. 
Eine Prophylaxe bzw. Behandlung im Falle einer Pandemie wäre sicherlich auch durch M2-Inhibitoren wie Amantadin und Rimantadin sowie durch Neuraminidase-Inhibitoren möglich [9]. Allerdings erscheint die Bereitstellung entsprechend großer Mengen aus ökonomischen und logistischen Gründen nicht realistisch. Insofern muss im Fall einer Pandemie auch an die Möglichkeiten der Übertragungsverhinderung gedacht werden. Über die Effektivität solcher Maßnahmen, wie z.B. die Schließung von Schulen, Universitäten und, wo immer möglich, anderen Institutionen, in denen in großem Umfang Personenkontakte stattfinden, ist nichts Eindeutiges bekannt. Prophylaktische Maßnahmen zur Verhinderung der bakteriellen Superinfektion stehen nicht zur Verfügung.

\section{Diagnostik der Virusgrippe}

Für die Diagnostik der Virusgrippe steht eine Fülle labortechnischer Verfahren zur Verfügung. Neben dem Nachweis des Virus in Zellkulturen gibt es über entsprechende Seren die Möglichkeit der Charakterisierung der Oberflächenantigene Neuraminidase und Hämagglutinin. Modifikationen von Nukleinsäure-amplifizierenden Techniken (RT-PCR) ermöglichen zwar einen raschen Nachweis und eine Charakterisierung der Influenza-Stämme [10]; sie stehen jedoch für den klinischen Alltag nur in sehr begrenztem Umfang zur Verfügung. Dies gilt auch für schnell einsetzbare und nach kurzer Zeit Ergebnisse liefernde immunologische Nachweisverfahren $[3,11]$. Ein weiterer Nachteil der meisten Laborverfahren ist die begrenzte Sensitivität und Spezifität bei nicht sehr schwer erkrankten Patienten.

Die Aufarbeitung eines Influenza-Stammes bis zu seiner endgültigen Charakterisierung unter Einschluss der molekularen Oberflächenantigene ist daher nach wie vor ein zeitaufwändiges und kostenintensives Verfahren. Gesicherte Fortschritte für die rasche Diagnostik im Bereich der Praxis oder des Krankenhauses haben sich in den letzten Jahren nicht ergeben.

Die Diagnostik der Virusgrippe beruht daher immer noch auf dem klinischen Urteil der entsprechenden Ärztin oder des Arztes. Dabei hat sich gezeigt, dass die Bewertung der klassischen Symptome einer Influenza-Grippe (hohes Fieber, Husten, Kopfund Gliederschmerzen, allgemeine Abgeschlagenheit) im Zusammenhang mit der Kenntnis der epidemiologischen Situation eine sehr hohe diagnostische Treffsicherheit hat $[12,13]$.

\section{Therapie der Influenza-A- und -B-Virus-Infektion}

\section{Antivirale Medikamente}

Für die medikamentöse Therapie der Influenza stehen die M2-Inhibitoren Amantadin und Rimantadin und die Neuraminidase-Inhibitoren Zanamivir und Oseltamivir zur Verfügung $[3,9]$. Die Verschreibung antiviral-wirksamer Medikamente bei der Influenza-Grippe erfolgt in der Regel auf dem Boden der klinischen Diagnose und der Kenntnis der epidemiologischen $\mathrm{Si}-$ tuation $[3,13]$.

\section{Amantadin und Rimantadin Wirkungsmechanismus}

Amantadin und Rimantadin sind verwandte antiviral-wirksame Medikamente, die ausschließlich gegenüber Influenza-A-Viren wirksam sind. Das Wirkungsprinzip beruht auf der Blockierung eines M2-Protein-Ionenkanals, der in der Virusmembran lokalisiert ist [3]. Durch diesen Kanal wandern während der Virusreplikation $\mathrm{H}^{+}$-Ionen, die für ein saures intravirales Milieu sorgen und hierdurch die Dissoziation des M1-Proteins vom Ribonukleoprotein-Komplex ermöglichen. Erst danach kann das Ribonukleoprotein in den Zellkern eindringen und seine Replikation beginnen.

\section{Pharmakokinetik}

Amantadin wird schnell und komplett resorbiert. Die Plasmaspitzenspiegel werden nach 2 Stunden erreicht. Die Spitzenkonzentrationen sind bei älteren Personen in der Regel um 50\% höher als bei jüngeren Personen. Ursächlich hierfür ist u.U. ein Unterschied in der Nierenleistungsfähigkeit, da die Substanz komplett renal ausgeschieden wird. Rimantadin wird ebenfalls gut, jedoch nicht so schnell wie Amantadin resorbiert. Die Spitzenplasmakonzentrationen werden in der Regel erst nach 4 Stunden erreicht. Rimantadin wird teilweise metabolisiert, die Metaboliten werden ebenfalls renal ausgeschieden. Hierdurch ergeben sich deutlich längere Halbwertszeichen als für das Amantadin.

\section{Dosierung und unerwïnschte Wirkungen}

Die Dosierungen von Amantadin und Rimantadin sind gleich. Kinder unterhalb des 9. Lebensjahres erhalten $5 \mathrm{mg}$ pro kg Körpergewicht, Erwachsene $1 \times$ täglich $200 \mathrm{mg}$ (bei normaler Nierenfunktion) und Personen oberhalb des 65 . Lebensjahres $1 \times$ täglich $100 \mathrm{mg}$.

Die wichtigsten unerwünschten Wirkungen des Amantadin sind Reaktionen des zentralen Nervensystems. Die Substanz kann zu Angstgefühlen, Depressionen, Schlaflosigkeit und in seltenen Fällen auch zu Halluzinationen und Krampfanfällen führen [14]. Ursächlich ist eine Freisetzung von Katecholaminen. Da Rimantadin diese Katecholamin-Freisetzung nicht induziert, treten neurologisch-psychiatrische Nebenwirkungen bei Rimantadin deutlich seltener auf [15].

Beide Substanzen haben hingegen mäßige gastro-intestinale Nebenwirkungen. Deutlich höhere Nebenwirkungsraten finden sich bei Patienten mit eingeschränkter Niereninsuffizienz. Auf die Nierenfunktion ist bei der Verordnung also streng zu achten.

\section{Effektivität}

Sowohl Amantadin als auch Rimantadin sind in der Prävention der Influenza-A-Infektion effektiv. Sie mindern auch die Rate der schweren Erkrankungen. In der Regel wird davon ausgegangen, dass der Prophylaxe-Effekt für die Infektion ca. 50\% und für die Erkrankung $70-90 \%$ beträgt $[16,17]$. Beide Substanzen eignen sich auch zur Behandlung der Influenza-Erkrankung. Unterschiede hinsichtlich ihrer Effektivität ergeben sich nicht $[18,19]$.

\section{Resistenzentwicklung}

Durch Mutationen am M2-Kanal entsteht eine Resistenz der Influenza-A-Viren [20]. Es besteht zwischen beiden Substanzen komplette Kreuzresistenz. Unter Behandlung entwickelt sich die 
Resistenz relativ rasch und kann bei 25 - 35\% der Stämme erwartet werden [21]. Diese Resistenzentwicklung hat bisher jedoch nur vereinzelt zur Verbreitung resistenter Virusstämme geführt. Insbesondere unter dem Aspekt des Einsatzes von Amantadin und Rimantadin bei einer Pandemie ist die Resistenzentwicklung jedoch im Auge zu behalten. Influenza-B-Viren sind immer resistent.

\section{Zanamivir und Oseltamivir Wirkungsmechanismus}

Zanamivir und Oseltamivir sind verwandte antivirale Substanzen mit Wirksamkeit gegen Influenza A und B, die beide den gleichen Wirkungsmechanismus aufweisen. Indem sie die virale Neuraminidase inhibieren, hindern sie das Virus einerseits nach dem Anheften die Zellmembran zu penetrieren, andererseits inhibieren sie die Ausschleusung der Viren, die nach der Penetration der Zellmembran von innen nach außen noch an zellulären $\mathrm{N}$-Acetyl-Neuraminsäurestrukturen haften, von denen sie gelöst werden müssen, ehe sie einen neuen Infektionszyklus in Gang setzen können [3].

\section{Pharmakokinetik}

Zanamivir steht lediglich zur Inhalation zur Verfügung. Dieses Applikationsprinzip ist für die relativ geringe Bioverfügbarkeit von $10-15 \%$ der inhalierten Dosis verantwortlich. Allerdings sind nach In-vitro-Untersuchungen die erreichbaren Konzentrationen in den respiratorischen Sekreten ausreichend, um klinisch wirksam zu sein.

Oseltamivir wird oral als Tablette oder Suspension verabreicht. Die Resorptionsrate beträgt 75\%. Oseltamivir ist eine pro-drug, die hepatisch zu Oseltamivir-Carboxylat umgebaut wird. Oseltamivir wird renal eliminiert, so dass eine Reduktion bei schwerer Niereninsuffizienz notwendig ist.

\section{Dosierung und unerwïnschte Wirkungen}

Die Dosierung von Zanamivir zur Therapie der Influenza-Erkrankung beträgt $20 \mathrm{mg}$ für Kinder, Erwachsene und ältere Personen ( $2 \times$ täglich $10 \mathrm{mg}$ per inhalationem). Zur Prophylaxe werden einmal täglich $10 \mathrm{mg}$ Zanamivir gegeben. Die einmal täglich einzunehmende Dosis von Oseltamivir beträgt bei Erwachsenen in der Therapie $150 \mathrm{mg}$ und wird bei Kindern nach dem Körpergewicht dosiert. Die Dosis bei der Prophylaxe beträgt $75 \mathrm{mg}$.

Die Nebenwirkungsfrequenz von Zanamivir ist gering und liegt vermutlich nicht wesentlich über der eines Plazebo-Präparates. Bei hoher bronchialer Hyperreagibilität muss u.U. an die Möglichkeit einer entsprechenden Prophylaxe durch kurzwirksame $\beta_{2}$-Mimetika gedacht werden.

Oseltamivir zeigt gastrointestinale Unverträglichkeiten bei ca. $10 \%$ der behandelten Personen. Die Verträglichkeit ist besser, wenn die Substanz zusammen mit Nahrung eingenommen wird. Weitere schwerwiegende unerwünschte Wirkungen sind für beide Substanzen bisher nicht bekannt.

\section{Effektivität}

Zanamivir und Oseltamivir haben ihre Wirksamkeit sowohl bei der Behandlung der Influenza als im Rahmen der Prophylaxe bewiesen. Allerdings zeigt sich ein therapeutischer Nutzen nur, wenn sehr frühzeitig nach Beginn der Symptomatik die Behandlung begonnen wird [22]. Bei Patienten, die innerhalb von 2 Tagen nach Erkrankungsbeginn behandelt werden, kann eine Reduktion der Krankheitstage von 1 bis 1,5 Tagen erreicht werden [23-25]. Darüber hinaus vermindern sich Grippe-spezifische Komplikationen wie Sinusitis, Otitis media, purulente Bronchitis und der Antibiotika-Verbrauch während einer Grippeerkrankung. Im Rahmen der Prophylaxe konnte gezeigt werden, dass die Gabe von Zanamivir per inhalationem [26,27] oder Oseltamivir oral [28] eine gute Wirksamkeit zeigt, wenn die Substanzen vor Beginn einer Epidemie eingenommen werden. Die Infektionsverhinderungsrate liegt bei $30-50 \%$, die Rate der Verhinderung einer Erkrankung bei 67 bzw. 84\% [29,30].

\section{Resistenzentwicklung}

Bisher liegen relativ wenige Daten über eine Resistenzentwicklung vor. Im Moment ist davon auszugehen, dass 1-2\% der Influenza-A- und -B-Stämme eine Resistenz gegenüber den Neuraminidaseinhibitoren aufweisen. Wahrscheinlich besteht aufgrund des identischen Wirkungsprinzips komplette Kreuzresistenz zwischen beiden Substanzen.

\section{Empfehlungen zur antiviralen Therapie und Prophylaxe bei der Influenza}

Der Einsatz antiviral-wirksamer Medikamente bei der InfluenzaGrippe ist hinsichtlich der Indikationsstellung nicht einfach zu bewerten. Die prinzipielle Schwierigkeit ergibt sich dabei aus der relativen Unsicherheit in der Diagnosestellung.

In Fällen einer klinisch schwerwiegenden Influenza-Grippe oder bei einer Grippe, die sich in Hochrisiko-Patienten entwickelt, liegt bei frühzeitigem Therapiebeginn vermutlich eine klare Indikation vor. Da Amantadin und Rimantadin nur gegenüber Influenza-A-Viren wirksam sind, muss bei Verordnung dieser Substanzen die epidemiologische Grundsituation bekannt sein. Im Gegensatz hierzu sind Zanamivir und Oseltamivir gegenüber Influenza-A- und -B-Stämmen wirksam.

Ob für antivirale Substanzen eine Indikation bei milden oder mäßigen Grippeerkrankungen besteht, kann zum jetzigen Zeitpunkt nicht eindeutig entschieden werden. Die nicht unbeträchtlichen Therapiekosten und die Möglichkeit einer weiteren Resistenzentwicklung müssen hier gegenüber dem erwartbaren Gewinn der Verkürzung der Krankheitsdauer um 1 bis 1,5 Tage sorgfältig abgewogen werden.

Eine Indikation zur prophylaktischen Verschreibung antiviralwirksamer Substanzen besteht mit hoher Wahrscheinlichkeit bei ungeimpften Personen mit einem hohen Risiko für einen komplizierten Influenzaverlauf, wenn die epidemiologische Situation eindeutig ist. Dies gilt auch für geimpfte Personen mit hohem Risiko, wenn die Übereinstimmung zwischen dem epidemischen Virusstamm und den in der Vakzine enthaltenen Virusstämmen schlecht ist. Eine weitere Indikation für eine prophy- 
laktische Therapie stellen Patienten mit definierter Immunsuppression dar. Möglicherweise kann beim Vorliegen weiterer Daten auch eine Indikation für Mitarbeiterinnen und Mitarbeiter im Gesundheitswesen und in Pflegeinstitutionen bestehen, wenn eine entsprechende epidemiologische Situation vorliegt. Eine besondere Bedeutung kommt den verfügbaren antiviralen Substanzen sicherlich im Fall einer neuen Pandemie zu. Hier ist jedoch die weltweite Versorgung mit den Substanzen vermutlich aus logistischen und ökonomischen Gründen nur schwer zu gewährleisten.

Das Vorhandensein antiviral-wirksamer Substanzen gegenüber Influenza A und B darf aber in keinem Fall dazu führen, dass die hoch effektiven Schutzimpfungsprogramme vernachlässigt werden, da sie immer noch den weitestgehenden Schutz gegenüber Grippeepidemien gewährleisten.

\section{Grippeschutzimpfung}

Für die Prävention stehen Grippeschutzimpfungen mit inaktivierten Spaltvakzinen oder attenuierten Lebendvakzinen zur Verfügung.

\section{Inaktivierte Spaltvakzine}

Die inaktivierte Spaltvakzine wird aus Viren hergestellt, die in Hühnerembryonen angezüchtet werden. Sie enthält als Standard $15 \mu \mathrm{g}$ des gewünschten Hämagglutinins. Die Zusammenstellung der Hämagglutinin- und Neuraminidase-Antigen-Komponenten erfolgt jährlich in Zusammenarbeit mit der Weltgesundheitsorganisation [3].

Wenn die Übereinstimmung zwischen der Spaltvakzine und dem Epidemie-Virusstamm hoch ist, beträgt der Effektivität der Influenza-Vakzination bei gesunden Erwachsenen $70-100 \%$. Ist die Übereinstimmung geringer, so sinkt die Effektivität der Impfung auf 30-60\%. Eine geringere Schutzwirkung gegenüber der Infektion ist auch bei jungen Kindern und älteren Menschen bekannt. Bei älteren Menschen besteht jedoch trotz des nicht optimalen Infektionsschutzes eine gute Schutzwirkung gegenüber komplizierten Verläufen der Influenza-Virusgrippe [31]. Eine Reihe von Studien hat in den vergangenen Jahren zeigen können, dass bei über 60-jährigen Patienten die Reduktion der Hospitalisierungsrate von geimpften gegenüber nicht geimpften Personen für die Diagnose „akute“ oder „chronische respiratorische Erkrankung“ zirka 30\% betrug, für die Diagnose „Lungenentzündung“ oder „Influenza-Virusgrippe“ zirka 40\% und für die Hospitalisierung wegen Herzinsuffizienz 25\%. Die Gesamtzahl der „Todesfälle aus allen Gründen“ wurde in der geimpften Population um zirka $50 \%$ gesenkt [31 - 39].

Die Ergebnisse bei gesunden Menschen zwischen dem 18. und 64. Lebensjahr haben in den Effektivitätsbeurteilungsstudien andere Zielkriterien gehabt. Hier gelang es jedoch, die Rate der Infektionen des oberen Respirationstraktes um $25 \%$ zu senken, die Anzahl der Arztbesuche wegen respiratorischer Infektionen um 44\% und die Anzahl der Erkrankungsfälle wegen oberer respiratorischer Infektionen um $43 \%$ zu vermindern [40,41]. Insgesamt kann bei jungen gesunden Erwachsenen durch die InfluenzaSchutzimpfung eine Reduktion der Erkrankungstage um 36\% erreicht werden [42].
Insofern steht außer Zweifel, dass die Impfung hoch protektiv ist $[41,43,44]$.

Die Impfung, bei der in der Regel 0,5 ml der Spaltvakzine $1 \times$ jährlich i.m. appliziert wird, ist gut verträglich. Zirka 25\% der Erwachsenen berichten über einen leichten Schmerz an der Injektionsstelle. Schwerwiegende Reaktionen sind jedoch ausgesprochen selten. Systemische Reaktionen haben in allen Studien das Niveau der Plazebo-Reaktionen nicht überschritten [40,45]. Die einzige Kontraindikation besteht in einer Hühnereiweißallergie. Diese ist stets zu erfragen. Die 1976 beobachtete Assoziation zwischen Guillain-Barré-Syndromen und der Influenza-Schutzimpfung hat sich in späteren Jahren nicht mehr herstellen lassen. Insgesamt ist davon auszugehen, dass auf 1 Millionen geimpfte Personen nicht mehr als ein Guillain-Barré-Syndrom auftritt $[3,46]$. Somit ist das Risiko für alle Altersgruppen bedeutend niedriger als das Risiko schwerer Influenza-Komplikationen.

Der Schutzeffekt der Impfung setzt etwa nach 10 Tagen ein. Die Influenza-Schutzimpfung induziert wegen der jährlichen Antigenveränderung der Viren nur eine Schutzwirkung für die aktuelle Saison [47].

Die Spaltvakzine-Impfung hat in den vergangenen Jahren eine deutlich weitere Verbreitung gefunden. In den USA wird geschätzt, dass 70\% der über 65-jährigen geimpft werden [48]. Die für Deutschland vorhandenen Daten zeigen, dass die Impfrate bei den über 60-jährigen in der Bundesrepublik Deutschland mit zirka 50\% bedeutend niedriger liegt. Sowohl in Europa als auch in den USA ist die Impfrate von Risikopersonen (Personen mit chronischen Erkrankungen) unterhalb des 60. Lebensjahres unbefriedigend [49]. Dies gilt auch für die Personen, die im Bereich des Gesundheitswesens oder in der Pflege tätig sind. Eine Anhebung der Impfrate in diesen beiden Gruppen hat neben dem individualmedizinischen Nutzen vor allem Bedeutung durch die Verminderung möglicher Vektoren, die die Infektion innerhalb von Arztpraxen, Hospitälern oder Pflegeeinrichtungen weiter verbreiten [49-53].

\section{Attenuierte Lebendvirus-Vakzine}

In den vergangenen Jahren ist eine attenuierte Lebendvirus-Vakzine hergestellt worden. Diese ist gegenüber Influenza A und Influenza B wirksam. Die Vakzine wird gentechnologisch hergestellt und kann intranasal als Spray verabreicht werden [54]. Bei ca. $15 \%$ der Geimpften kommt es zu milden Symptomen im Bereich des oberen Respirationstraktes. Die Effektivität der Lebendvakzine ist sowohl in vitro durch entsprechende Anstiege von Antikörpern [55] als auch in vivo hinsichtlich ihrer Schutzwirkung gegenüber klinisch fassbaren Erkrankungen geprüft worden. Bisher wurden jedoch überwiegend Kinder und junge gesunde Erwachsene untersucht [40,56-59]. In diesen Gruppen ist die Effektivität der Lebendvakzine nahezu identisch zu der intramuskulär zu verabreichenden Spaltvakzine. Es ist daher damit zu rechnen, dass in naher Zukunft mit der Lebendvakzine eine Substanz zur Verfügung steht, die ihre Indikation insbesondere bei Kindern finden wird. 
Impfempfehlungen

Die internationalen Empfehlungen zur Influenza-Schutzimpfung sind weitgehend identisch. Die Ständige Impfkommission des Bundesgesundheitsamts (STIKO) empfiehlt die InfluenzaSchutzimpfung [51]:

- bei allen Personen im Alter von mehr als 60 Jahren (Standardimpfung);

- bei Kindern, Jugendlichen und Erwachsenen mit erhöhter gesundheitlicher Gefährdung infolge eines Grundleidens wie z. B. chronische Lungen-, Herz-Kreislauf-, Leber- und Nierenkrankheiten, Diabetes und anderen Stoffwechselkrankheiten, Immundefizienz, HIV-Infektion - sowie bei Bewohnern von Alters- oder Pflegeheimen (Indikationsimpfung);

- bei allen Personen mit erhöhter Gefährdung, z. B. bei medizinischem Personal, bei Personen in Einrichtungen mit umfangreichem Publikumsverkehr sowie bei Personen, die als mögliche Infektionsquellen für die von ihnen betreuten ungeimpften Risikopersonen fungieren können (Indikationsimpfung, Impfung bei beruflichem Risiko);

- bei allen Personen, wenn Epidemien auftreten oder aufgrund epidemiologischer Beobachtungen befürchtet werden (Indikationsimpfung).

Von besonderer Bedeutung ist die Tatsache, dass geimpfte Personen sich nicht nur selbst schützen, sondern auch einen wichtigen Beitrag zur Vermeidung von Epidemien leisten, da die geimpften Personen im Fall einer Epidemie den Erreger nicht oder nur in deutlich reduziertem Ausmaß weiterverbreiten.

\section{Literatur}

${ }^{1}$ Schaberg T, Kaufmann S. Infektion. In: Siegenthaler W, Hrsg. Klinische Pathophysiologie. Stuttgart: Thieme, 2001: 565-590

${ }^{2}$ Nicholson KG. Human influenza. In: Nicholson KG, Webster RG, Hay AJ, editors. Textbook of influenza. Oxford: Blackwell Science, 1998: 219-264

${ }^{3}$ Couch RB. Prevention and treatment of influenza. N Engl J Med 2002; 343: $1778-1787$

${ }^{4}$ Hak E, Verheij TJ, van Essen GA et al. Prognostic factors for influenzaassociated hospitalization and death during an epidemic. Epidemiol Infect 2001; 126(2): $261-268$

${ }^{5}$ Robert Koch-Institut. Vorbereitung auf die Influenza-Saison 2001/2002. Epidemiol Bull 2001; 36/2001: 271-273

${ }^{6}$ Robert Koch-Institut. Zur Surveillance der Influenza in Deutschland. Epidemiol Bull 2001; 36/2001: 274-275

${ }^{7}$ Robert Koch-Institut. Erste Einschätzung der Influenza-Saison 2001/2002. Epidemiol Bull 2002; 17/2002: 135-136

${ }^{8}$ Fock R, Bergmann H, Bußmann $\mathrm{H}$ et al. Influenza pandemic: preparedness planning in Germany. Euro Surveillance 2002; 7(1): 1 -5

${ }^{9}$ Wutzler P. Aktuelle Probleme bei der Influenza-Bekämpfung. Chemotherapie J 2002; 11: 154-156

${ }^{10}$ Atmar RL, Baxter BD, Dominguez EA et al. Comparison of reverse transcription-PCR with tissue culture and other rapid diagnostic assays for detection of type A influenza virus. J Clin Microbiol 1996; 34: $2604-2606$

${ }^{11}$ Noyola DE, Clark B, O'Donnell FT et al. Comparison of a new neuraminidase detection assay with an enzyme immunoassay, immunofluorescence, and culture for rapid detection of influenza $A$ and $B$ viruses in nasal wash specimens. J Clin Microbiol 2000; 38(3): 1161 - 1165

12 Monto AS, Gravenstein S, Elliott M et al. Clinical signs and symptoms predicting influenza infection. Arch Intern Med 2000; 160(21): $3243-3247$
${ }^{13}$ van Elden LJ, van Essen GA, Boucher CA et al. Clinical diagnosis of influenza virus infection: evaluation of diagnostic tools in general practice. Br J Gen Pract 2001; 51(469): 630-634

${ }^{14}$ Bryson YJ, Monahan C, Pollack M et al. A prospective double-blind study of side effects associated with the administration of amantadine for influenza A virus prophylaxis. J Infect Dis 1980; 141(5): 543-547

${ }^{15}$ Hayden FG, Sable CA, Connor JD et al. Intravenous ribavirin by constant infusion for serious influenza and parainfluenzavirus infection. Antivir Ther 1996; 1(1): $51-56$

${ }^{16}$ Karlsson M, Reichard O, Linde A et al. Amantadine for prophylaxis against influenza A. Scand J Infect Dis 1987; 19(1): 141-142

${ }^{17}$ Dolin R, Reichman RC, Madore HP et al. A controlled trial of amantadine and rimantadine in the prophylaxis of influenza $\mathrm{A}$ infection. $\mathrm{N}$ Engl J Med 1982; 307(10): 580-584

${ }^{18}$ Jefferson T, Demicheli V, Rivetti D et al. Cochrane reviews and systematic reviews of economic evaluations. Amantadine and rimantadine in the prevention and treatment of influenza. Pharmacoeconomics 1999; 16 Suppl 1: $85-89$

${ }^{19}$ Jefferson TO, Demicheli V, Deeks JJ et al. Amantadine and rimantadine for preventing and treating influenza $\mathrm{A}$ in adults. In: The Cochrane Library, 4. Oxford: Update Software, 2001

${ }^{20}$ Hayden FG, Belshe RB, Clover RD et al. Emergence and apparent transmission of rimantadine-resistant influenza A virus in families. N Engl J Med 1989; 321(25): 1696-1702

${ }^{21}$ Masuda $\mathrm{H}$, Suzuki H, Oshitani $\mathrm{H}$ et al. Incidence of amantadine-resistant influenza A viruses in sentinel surveillance sites and nursing homes in Niigata, Japan. Microbiol Immunol 2000; 44(10): 833-839

${ }^{22}$ Jefferson T, Demicheli V, Deeks J et al. Neuraminidase inhibitors for preventing and treating influenza in healthy adults. In: The Cochrane Library, 4. Oxford: Update Software, 2001

${ }^{23}$ Hayden FG, Osterhaus AD, Treanor JJ et al. Efficacy and safety of the neuraminidase inhibitor zanamivir in the treatment of influenzavirus infections. GG167 Influenza Study Group. N Engl J Med 1997; 337(13): $874-880$

24 Treanor JJ, Hayden FG, Vrooman PS et al. Efficacy and safety of the oral neuraminidase inhibitor oseltamivir in treating acute influenza: a randomized controlled trial. US Oral Neuraminidase Study Group. JAMA 2000; 283(8): 1016-1024

${ }^{25}$ Monto AS, Fleming DM, Henry D et al. Efficacy and safety of the neuraminidase inhibitor zanamivirin the treatment of influenza A and B virus infections. J Infect Dis 1999; 180(2): 254-261

${ }^{26}$ Hayden FG, Gubareva LV, Monto AS et al. Inhaled zanamivir for the prevention of influenza in families. Zanamivir Family Study Group. N Engl J Med 2000; 343(18): 1282 - 1289

${ }^{27}$ Monto AS, Robinson DP, Herlocher ML et al. Zanamivir in the prevention of influenza among healthy adults: a randomized controlled trial. JAMA 1999; 282(1): $31-35$

${ }^{28}$ Hayden FG, Atmar RL, Schilling M et al. Use of the selective oral neuraminidase inhibitor oseltamivir to prevent influenza. N Engl J Med 1999; 341(18): 1336-1343

${ }^{29}$ Parker R, Loewen N, Skowronski D. Experience with oseltamivir in the control of a nursing home influenza B outbreak. Can Commun Dis Rep $2001 ; 27(5): 37-40$

${ }^{30}$ Bowles SK, Lee W, Simor AE et al. Use of oseltamivir during influenza outbreaks in Ontario nursing homes, 1999-2000. J Am Geriatr Soc 2002; 50(4): 608-616

${ }^{31}$ Nichol KL, Wuorenma J, Von Sternberg T. Benefits of influenza vaccination for low-, intermediate-, and high-risk senior citizens. Arch Intern Med 1998; 158: 1769-1776

32 Deguchi Y, Takasugi Y, Tatara K. Efficacy of influenza vaccine in the elderly in welfare nursing homes: reduction in risks of mortality and morbidity during an influenza A (H3N2) epidemic. J Med Microbiol 2000; 49(6): $553-556$

${ }^{33}$ Nichol KL, Margolis KL, Wuorenma J et al. The efficacy and cost effectiveness of vaccination against influenza among elderly persons living in the community. N Engl J Med 1994; 331: 778-784

${ }^{34}$ Christenson B, Lundbergh P, Hedlund J et al. Effects of a large-scale intervention with influenza and 23 -valent pneumococcal vaccines in adults aged 65 years or older: a prospective study. Lancet 2001; 357(9261): $1008-1011$

${ }^{35}$ Nordin J, Mullooly J, Poblete $S$ et al. Influenza vaccine effectiveness in preventing hospitalizations and deaths in persons 65 years or older in Minnesota, New York, and Oregon: data from 3 health plans. J Infect Dis 2001; 184(6): 665-670 
${ }^{36}$ Demicheli V, Rivetti D, Deeks JJ et al. Vaccines for preventing influenza in healthy adults. Cochrane Database Syst Rev 2001; (4): CD001269

${ }^{37}$ Nichol KL, Margolis KL et al. Effectiveness of influenza vaccine in the elderly. Gerontology 1996; 42(5): 274-279

38 Nichol KL, Baken L, Nelson A. Relation between influenza vaccination and outpatient visits, hospitalization, and mortality in elderly persons with chronic lung disease. Ann Intern Med 1999; 130(5): 397- 403

${ }^{39} \mathrm{Nichol} \mathrm{KL}$, Goodman M. The health and economic benefits of influenza vaccination for healthy and at-risk persons aged 65 to 74 years. Pharmacoeconomics 1999; 16 Suppl 1: $63-71$

${ }^{40} \mathrm{Nichol} \mathrm{KL}$, Margolis KL, Lind A et al. Side effects associated with influenza vaccination in healthy working adults. A randomized, placebocontrolled trial. Arch Intern Med 1996; 156: 1546-1550

${ }^{41}$ Bridges CB, Thompson WW, Meltzer MI et al. Effectiveness and costbenefit of influenza vaccination of healthy working adults: A randomized controlled trial. JAMA 2000; 284(13): 1655-1663

${ }^{42}$ Leighton L, Williams M, Aubery D et al. Sickness absence following a campaign of vaccination against influenza in the workplace. Occup Med (Lond) 1996; 46(2): 146-150

43 Postma MJ, Jansema P, van Genugten ML et al. Pharmacoeconomics of influenza vaccination for healthy working adults: reviewing the available evidence. Drugs 2002; 62(7): $1013-1024$

${ }^{44} \mathrm{Nichol} \mathrm{KL}$. Cost-benefit analysis of a strategy to vaccinate healthy working adults against influenza. Arch Intern Med 2001; 161(5): $749-759$

${ }^{45}$ Allsup SJ, Gosney M, Regan M et al. Side effects of influenza vaccination in healthy older people: a randomised single-blind placebo-controlled trial. Gerontology 2001; 47(6): 311 - 314

${ }^{46}$ Lasky T, Terracciano GJ, Magder L et al. The Guillain-Barré syndrome and the $1992-1993$ and $1993-1994$ influenza vaccines. N Engl J Med 1998; 339: $1797-1802$

${ }^{47}$ Beyer WE, de Bruijn IA, Palache AM et al. Protection against influenza after annually repeated vaccination: a meta-analysis of serologic and field studies. Arch Intern Med 1999; 159(2): 182 - 188

48 Centers for Disease Control and Prevention. Influenza and pneumococcal vaccination levels among adults aged $>$ or $=65$ years - United States, 1997. JAMA 1998; 280: $1818-1819$

${ }^{49}$ Robert Koch-Institut. Teilnahme an Influenza- und PneumokokkenSchutzimpfung. Epidem Bull 2002; 16/2002: 127-131
${ }^{50}$ Nichol KL, Hauge M. Influenza vaccination of healthcare workers. Infect Control Hosp Epidemiol 1997; 18(3): 189-194

${ }^{51}$ STIKO. Empfehlungen der Ständigen Impfkommission (STIKO) am Robert Koch-Institut/Stand Juli 2002. Epidemiol Bull 2002; 28: 227-242

52 Wilde JA, McMillan J, Serwint J et al. Effectiveness of influenza vaccine in health care professionals: a randomized trial. JAMA 2002; 281: $908-913$

${ }^{53}$ Robert Koch-Institut. Influenza-Schutzimpfung im Rahmen einer Aktion angeboten. Epidemiol Bull 2002; 38/2002: 321 - 323

${ }^{54}$ Kiderman A, Furst A, Stewart B et al. A double-blind trial of a new inactivated, trivalent, intra-nasal anti-influenza vaccine in general practice: relationship between immunogenicity and respiratory morbidity over the winter of 1997-98. J Clin Virol 2001; 20(3): 155-161

55 Jackson LA, Austin G, Chen RT et al. Safety and immunogenicity of varying dosages of trivalent inactivated influenza vaccine administered by needle-free jet injectors. Vaccine 2001; 19(32): 4703 - 4709

${ }^{56}$ Mendelman PM, Cordova J, Cho I. Safety, efficacy and effectiveness of the influenza virus vaccine, trivalent, types $A$ and $B$, live, cold-adapted (CAIV-T) in healthy children and healthy adults. Vaccine 2001; 19(17-19): $2221-2226$

${ }^{57}$ Belshe RB, Gruber WC. Safety, efficacy and effectiveness of cold-adapted, live, attenuated, trivalent, intranasal influenza vaccine in adults and children. Philos Trans R Soc Lond B Biol Sci 2001; 356(1416): $1947-1951$

${ }^{58}$ Treanor JJ, Kotloff K, Betts RF et al. Evaluation of trivalent, live, coldadapted (CAIV-T) and inactivated (TIV) influenza vaccines in prevention of virus infection and illness following challenge of adults with wild-type influenza A (H1N1), A (H3N2), and B viruses. Vaccine 1999; 18(9-10): 899-906

${ }^{59}$ Longini IM, Halloran ME, Nizam A et al. Estimation of the efficacy of live, attenuated influenza vaccine from a two-year, multi-center vaccine trial: implications for influenza epidemic control. Vaccine 2000; 18(18): $1902-1909$

\section{Bereits publizierte Beiträge zu dieser Serie:}

${ }^{1}$ Diagnostik und Therapie der Legionellen-Pneumonie. Pneumologie 2002; 56: $695-703$ 